\title{
Anforderungen an den Einsatz von Digitalisierung und KI
}

\author{
Anwendungen zur Steigerung der Energieeffizienz \\ Dennis Bode, Henry Ekwaro-Osire, BIBA - Bremer Institut für Produktion und Logistik GmbH und \\ Klaus-Dieter Thoben, Universität Bremen/BIBA
}

\begin{abstract}
Energieeffizienz gewinnt eine immer stärkere Bedeutung in allen Branchen des produzierenden Gewerbes. Mit Technologien aus den Bereichen Digitalisierung und Künstlicher Intelligenz (KI) können innovative Lösungen für eine energieeffizientere Herstellung beispielsweise durch eine KI-gestützte Prozessführung einen entscheidenden Beitrag zur Nachhaltigkeit und zur Wettbewerbsfähigkeit eines Unternehmens leisten. Die Anforderungen an den Einsatz und die reibungslose Integration von $\mathrm{KI}$ in Unternehmensprozesse sind komplex und vielfältig. Verschiedene Beteiligte müssen bei der Erfassung der Anforderungen integriert und unterschiedliche Technologieaspekte und Geschäftsfelder beachtet werden. Gegenstand dieses Beitrags ist ein Vorgehen zur Erfassung dieser Anforderungen bei der Durchführung innovativer Digitalisierungs- und KI-Projekte zur Steigerung der Energieeffizienz im produzierenden Gewerbe.
\end{abstract}

\section{Anwendungen von KI \\ zur Steigerung der \\ Energieeffizienz}

Der Einsatz innovativer KI-Lösungen kann einen entscheidenden Beitrag zur Steigerung der Energieeffizienz produzierender Unternehmen leisten. Vor allem bei wechselnden Produktionsbedingungen bedingt durch schwankende Rohwarenqualität oder Umweltflüsse ist die kontinuierliche und dynamische Einstellung energieeffizienter Prozessparameter eine komplexe Aufgabe. Im Fokus der Unternehmen steht häufig vor allem die Sicherung der Produktqualität. Die datengetriebene Modellierung von Produktionsprozessen mit Kl-unterstützten Modellen und anschließender Optimierung bezüglich der Produktqualität und des Energieverbrauchs kann unterstützen, die Energieeffizienz der Produktion zu verbessern.

Auch weitere Einsatzgebiete wie die $\mathrm{Kl}$-gestützte Identifizierung von Energieverbrauchs-optimalen Wartungsvorgängen für Produktionsanlagen, die
Erzeugung einer Energiedatentransparenz oder die modellbasierte Vorhersage von Energieverbräuchen erfordern den Einsatz komplexer Ansätze der künstlichen Intelligenz und Digitalisierung.

Motivation für eine gründliche Anforderungsanalyse

Die erfolgreiche Anwendung von KI zur Steigerung der Energieeffizienz wird zu Beginn eines Projekts durch eine zielgerichtete Anforderungsanalyse ermöglicht. Insbesondere bei Lösungsansätzen in den Bereichen Digitalisierung und Kl ist dies wichtig, denn die Schnittstellen zu Mitarbeitenden und bestehender Infrastruktur sind hier besonders groß. Werden Bedürfnisse und technische Randbedingungen zu Beginn nicht beachtet und bei der Entwicklung nicht berücksichtigt, verringert dies den Nutzen und die Akzeptanz der entwickelten Lösung. Im schlechtesten Fall ist sogar der sinnvolle Einsatz im späteren Produktivbetrieb gefährdet. Vorhandene Energiesparpotenziale
Requirements for the Use of Digitization and Al Innovative digital and Al solutions for more energy-efficient production can decisively contribute to the environmental impact and competitiveness of companies, especially in the manufacturing industry. Requirements for the functionality and implementation of these solutions are complex and diverse; multiple stakeholders need to be addressed when eliciting requirements and various technology and business aspects have to be considered. This article presents a procedure for requirements elicitation for energy efficiency digitalization and $\mathrm{Al}$ projects.

Keywords:

artificial intelligence, machine learning, energy efficiency, digitalization, sustainable production, requirement engineering

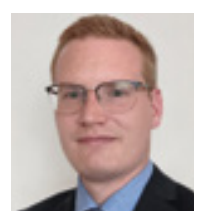

Dennis Bode, M. Sc. arbeitet als wissenschaftlicher Mitarbeiter im Forschungsbereich Informations- und kommunikationstechnische Anwendungen in der Produktion am BIBA.

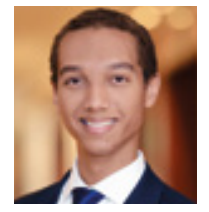

Henry Ekwaro-Osire, M. Eng. arbeitet als wissenschaftlicher Mitarbeiter im Forschungsbereich Informations- und kommunikationstechnische Anwendungen in der Produktion am BIBA.

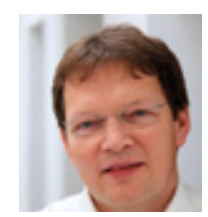

Prof. Dr.-Ing. Klaus-Dieter Thoben ist Professor für Integrierte Produktentwicklung an der Universität Bremen und Mitglied der Geschäftsführung des BIBA. 
können dann nicht wie geplant vollumfänglich ausgeschöpft werden.

Die Herausforderung bei der Anforderungsanalyse ist, dass die Mehrheit der Mitarbeitenden wenig oder keine Erfahrungen und Berührungspunkte mit KI vorweisen können. Das betrifft zum einen die Gruppe der Produktionsmitarbeitenden, die später mit der KI als Assistenz arbeiten sollen. Zum anderen haben dieses Defizit auch Führungskräfte, die auf Grundlage der angestrebten Lösung strategische Unternehmensentscheidungen treffen müssen. Im Gegensatz zu großen Unternehmen sind bei kleinund mittelständischen Unternehmen erschwerend häufig auch keine IT-Fachleute vorhanden, die spezialisiert auf den Einsatz von Digitalisierung und $\mathrm{KI}$ sind [1].

Dies führt zu der Herausforderung, dass die Mehrheit der eigenen Mitarbeitenden sehr starke Unterstützung bei der Definition von Anforderungen an Lösungen basierend auf $\mathrm{KI}$ und Digitalisierung benötigt. Denn durch das fehlende Verständnis der Funktionsweise ist auch die Vorstellungskraft bezüglich der Potenziale, Einschränkungen und Voraussetzungen beim Einsatz von KI gehemmt. Gerade diese Vorstellungskraft ist jedoch bei der Identifizierung von relevanten Anforderungen zwingend erforderlich. Die Anforderungen der Endnutzenden sind entscheidend für den Erfolg der Lösung und müssen daher gründlich aufgenommen werden.

Um dies zu unterstützen, wird in diesem Beitrag ein Vorgehen vorgestellt, das trotz der genannten Herausforderungen die Erstellung einer umfangreichen und detaillierten Anforderungssammlung ermöglicht.

\section{Bestehende Methoden zur Anforderungserfassung}

Requirements Engineering (RE) ist das Fachgebiet, das sich ausschließlich mit Anforderungen befasst. Der RE-Prozess besteht aus Erfassung, Analyse, Spezifikation, Validierung und Verifikation, und Verwaltung [2-4]. Dieser Beitrag behandelt die Erfassung insbesondere im Kontext von KI-Projekten. In der Literatur und Praxis sind bereits viele Erfassungsmethoden etabliert. Um das entwickelte Vorgehen der Autoren besser zu differenzieren, sind ausgewählte Vor- und Nachteile der gängigsten Methoden in Abbildung 1 gelistet und kategorisiert wie in [5].

Es sollte beachtet werden, dass jede Methode gewisse Vorteile in bestimmten Situationen bringt, und oft mehrere in Kombination verwendet wer- den, da jede Methode auch gewisse Nachteile hat [5]. Nach Evaluierung der Methoden, haben die Autoren festgestellt, dass keine im Einzelnen optimal für diesen Kontext geeignet ist. Somit haben die Autoren ein neues Vorgehen aus einer Kombination und Anpassung der obigen Methoden entwickelt, das auf die beschriebene Problemstellung angepasst wurde. Dieses Vorgehen ist im folgenden Kapitel erläutert.

\section{Vorstellung des entwickelten vorgehens}

Das entwickelte Vorgehen wird im Folgenden Schritt für Schritt vorgestellt und ist in Abbildung 2 dargestellt.

\section{Identifizierung von Sichtweisen und Rollen}

Wie bereits erwähnt ist die Gruppe der Beteiligten bei der Umsetzung von KI- und Digitalisierungsprojekten sehr vielfältig. Nach Analyse der Autoren müssen zumindest Entwickler*innen, Implementierer*innen und Nutzer*innen der zu entwickelnden Lösung einbezogen werden. Häufig können zudem innerhalb dieser übergeordneten Gruppierungen weitere unterschiedliche Rollen identifiziert werden. Alle diese Rollen haben eigene Aufgaben in Verbindung mit dem geplanten KI-Vorhaben und müssen in die Anforderungsanalyse einbezogen werden.

\section{Definition von Kategorien}

Nach dem Vorbild eines strukturierten Interviews werden im zweiten Schritt Kategorien definiert, für die Anforderungen gesammelt werden sollen. Auf die strikte Vorgabe von Fragen wird verzichtet, um den Anforderungsgeber*innen Flexibilität und Interpretationsspielraum der Kategorie für die eigenen Aufgaben einräumen zu können. Neben technischen Kategorien können hier auch betriebswirtschaftliche Aspekte oder Fragen der Nutzerfreundlichkeit betrachtet werden. Die Vorgabe von Kategorien ist aufgrund der häufig fehlenden Erfahrungen der Mitarbeitenden mit $\mathrm{KI}$-Lösungen notwendig. Sie soll die Diskussion breit öffnen und den Anforderungsgeber*innen eine Hilfestellung geben, welche Themenfelder bei der Nutzung der KI betrachtet werden.

\section{Durchführung eines Workshops}

Nach der Definition der Rollen und der Aufstellung der Themenfelder werden die Anforderungen im Rahmen eines Workshops aufgenommen. An diesem Workshop müssen alle definierten Rollen mindestens durch eine Person vertreten sein.

Da den Personen die direkte Formulierung von Anforderungen an die KI-Lösung aufgrund der 


\begin{tabular}{|c|c|c|c|}
\hline Kategorie & Methode & Vorteile & Nachteile \\
\hline \multirow{2}{*}{ 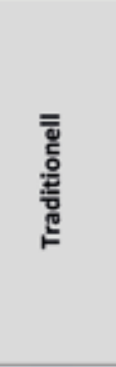 } & Interviews & $\begin{array}{l}\text { - Erfassung detaillierter Informationen } \\
\text { - Flexibler Ablauf } \\
\text { - Offene Fragen }\end{array}$ & $\begin{array}{l}\text { - Antworten müssen interpretiert werden } \\
\text { - Überwiegend qualitative Information }\end{array}$ \\
\hline & $\begin{array}{l}\text { Umfragen \& } \\
\text { Fragebögen }\end{array}$ & $\begin{array}{l}\text { - Befragung vieler Personen in relativ } \\
\text { kurzer Zeit } \\
\text { - Spezifische Informationen können gezielt } \\
\text { gesammelt werden }\end{array}$ & $\begin{array}{l}\text { - Begrenzung der Aussagen durch Vorgabe der } \\
\text { Fragen } \\
\text { - Teilnehmer*innen können Fragen anders } \\
\text { interpretieren }\end{array}$ \\
\hline \multirow{3}{*}{ 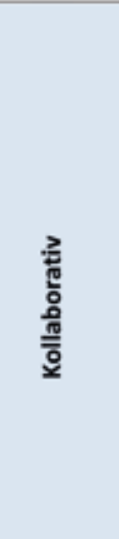 } & Focus Groups & $\begin{array}{l}\text { - Befragung mehrerer Stakeholder } \\
\text { gleichzeitig } \\
\text { - Erkenntnisse durch Interaktion zwischen } \\
\text { Stakeholdern }[6,7]\end{array}$ & $\begin{array}{l}\text { - Oft teuer oder nicht verfügbar } \\
\text { - Gefahr von }{ }_{n} \text { Groupthink" }\end{array}$ \\
\hline & Workshops & $\begin{array}{l}\text { - Interaktiv } \\
\text { - Erkenntnisse können gemeinsam mit } \\
\text { Nutzerinnen erarbeitet werden } \\
\text { - Mehrere Nutzer*innen involviert }\end{array}$ & $\begin{array}{l}\text { - Benötigt weitere Methoden in Kombination um } \\
\text { Anforderungen zu erheben }\end{array}$ \\
\hline & Brain-storming & $\begin{array}{l}\text { - Sammlung einer Vielfalt an Ideen } \\
\text { - Alle Teilnehmerinnen tragen bei }\end{array}$ & $\begin{array}{l}\text { - Moderation kann schwierig sein } \\
\text { - Wenig Tiefe } \\
\text { - Nachträgliche Aufbereitung notwendig }\end{array}$ \\
\hline ᄒ & Prototypen & $\begin{array}{l}\text { - Frūhe Sammlung von Erfahrungen mit } \\
\text { dem Produkt } \\
\text { - Nutzerln kann Feedback geben }\end{array}$ & $\begin{array}{l}\text { - Zeitintensiv/teuer } \\
\text { - Oft nicht ausreichend realisierbar }\end{array}$ \\
\hline \multirow{2}{*}{ 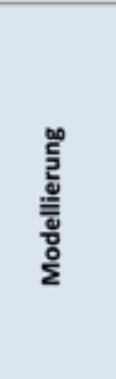 } & $\begin{array}{l}\text { Szenarien \& Use } \\
\text { Cases }\end{array}$ & $\begin{array}{l}\text { - Weniger aufwendiger Ersatz für } \\
\text { Prototyping } \\
\text { - Abbildung vieler unterschiedlicher } \\
\text { Situationen }\end{array}$ & $\begin{array}{l}\text { - Oft schwierig, die richtigen Szenarien zu } \\
\text { identifizieren }\end{array}$ \\
\hline & $\begin{array}{l}\text { Geschäfts- } \\
\text { prozesse }\end{array}$ & $\begin{array}{l}\text { - Deutliche Spezifizierung von } \\
\text { Geschăftsziele und -anforderungen. [8] } \\
\text { - Anforderungen können gezielt auf } \\
\text { Geschäftsprozesse zurūckgeführt werden }\end{array}$ & $\begin{array}{l}\text { - Hohes Domänenwissen erforderlich } \\
\text { - Detaillierte Modellerstellung ist zeitaufwendig }\end{array}$ \\
\hline 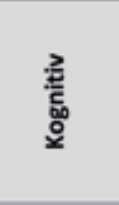 & Ontologien & $\begin{array}{l}\text { - Gut nachverfolgbare Darstellung von } \\
\text { Beziehungen zwischen den } \\
\text { Anforderungen } \\
\text { - Laufende Integration einer großen Menge } \\
\text { unterschiedlicher Anforderungen [9] }\end{array}$ & $\begin{array}{l}\text { - Aufwändige Erstellung, nicht besonders intuitiv } \\
\text { zu lesen }\end{array}$ \\
\hline 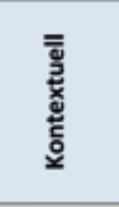 & $\begin{array}{l}\text { Ethnographie \& } \\
\text { Ethno- } \\
\text { methodologie }\end{array}$ & $\begin{array}{l}\text { - Besseres Verständnis der Beziehung } \\
\text { zwischen den Nutzer*innen } \\
\text { - Erfassung von Anforderungen die } \\
\text { Nutzer*innen sonst nicht erwāhnt hātten } \\
\text { [10] }\end{array}$ & $\begin{array}{l}\text { - Erzeugt eher Verstāndnis der Domäne als } \\
\text { konkrete Anforderungen [11] }\end{array}$ \\
\hline \multirow{3}{*}{ 高 } & User Stories & $\begin{array}{l}\text { - Ganzheitliches Verstãndnis der } \\
\text { Anforderung, nicht nur technisches } \\
\text { Verstăndnis }\end{array}$ & $\begin{array}{l}\text { - Ergebnisse mũssen in eine technische } \\
\text { Anforderung übersetzt werden }\end{array}$ \\
\hline & Mind Mapping & $\begin{array}{l}\text { - Bringt Struktur wo viele Ideen } \\
\text { durchmischt sind } \\
\text { - Gut geeignet für Verarbeitung von bereits } \\
\text { gesammelten Anforderungen }\end{array}$ & $\begin{array}{l}\text { - Beinhaltet meist nicht die Nutzer*innen } \\
\text { - Benötigt weitere Methoden in Kombination um } \\
\text { Anforderungen zu erheben }\end{array}$ \\
\hline & Storytelling & $\begin{array}{l}\text { - Flexibler als User Stories } \\
\text { - Nutzer*innen kōnnen auf-einander } \\
\text { Wissen aufbauen }\end{array}$ & $\begin{array}{l}\text { - Benötigt gründliche Vorbereitung um nutzbare } \\
\text { Ergebnisse zu garantieren }\end{array}$ \\
\hline
\end{tabular}

Bild 1: Überblick über Techniken zur Ermittlung von Anforderungen einschließlich ausgewählter Beispiele für Vor- und Nachteile. 
Bild 2: Grafische Darstellung der Schritte des Vorgehens anhand der Vorlage für die Anforderungs-Workshops.
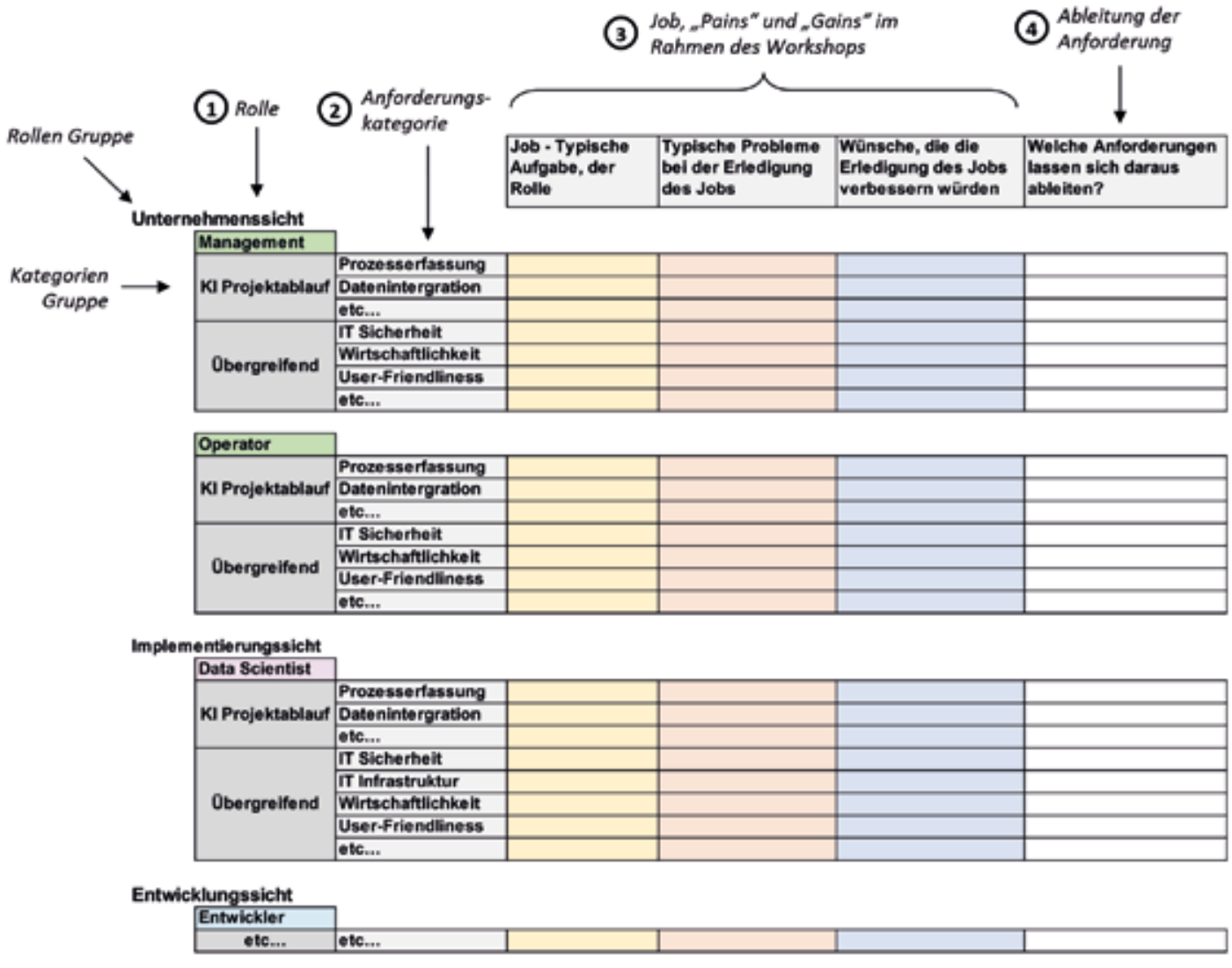

Ete..

mangelnden Erfahrung und des mangelnden Verständnisses der Funktionsweise häufig schwerfällt, werden die Anforderungen über eine Hilfestellung erhoben.

Vorbild sind Elemente der Methode des „Value Proposition Canvas" [12], die in Kombination mit den Kategorien und Rollen angewandt werden. Dabei werden zunächst für jede Rolle "Jobs" in den unterschiedlichen Kategorien definiert, die die Mitarbeiter*innen in Zusammenhang mit Digitalisierung und $\mathrm{KI}$ oder mit $\mathrm{KI}$-Unterstützung erledigen müssen. Für jeden „Job" überlegen die Mitarbeiter*innen, welche Schwierigkeiten („Pains") sie bei Erledigung dieses Jobs haben. Als dritter Schritt wird angegeben, welche Maßnahmen und Ideen zur Erleichterung oder Beschleunigung dieses Jobs helfen würden ("Gains"). Mit dieser stufenweisen Heranführung werden die Aspekte der eigentlichen Grundanforderungen schrittweise gemeinsam für die unterschiedlichen Rollen erhoben.

\section{Ableitung der Anforderungen für die KI/ Digitalisierungs-Lösungen}

Die resultierenden Anforderungen werden nach dem Workshop von KI-Expert*innen mit Kenntnissen aus dem RE auf Basis der "Jobs", „Pains" und "Gains" abgeleitet und definiert. Idealerweise sind die KI-Expert*innen zugleich Moderator*innen des Workshops. Der Grund für die
Ableitung der Anforderung nach dem Workshop ist, dass die Nutzenden nicht selbst KI-Expert*innen sind. Sie können daher nicht einschätzen, was die beste Lösung wäre. Das bekannte Zitat (oft unbewiesen Henry Ford zugeschrieben) ist hier treffend. Dieser hatte behauptet,wenn er seine Kunden gefragt hätte, was sie wollen, hätten sie gesagt, schnellere Pferde. Deswegen ist es bei der Ableitung wichtig, das Kernbedürfnis innerhalb der "Pain" und "Gain" zu identifizieren. Die Anforderungen sollen nicht lösungsorientiert definiert sein, sondern bedürfnisorientiert. Somit wird vermieden, den Lösungsraum zu früh einzugrenzen.

\section{Verwaltung der gesammelten Anforderungen} Die Anforderungen können über bestehende Systeme zum Anforderungsmanagement gespeichert werden. Sinnvoll ist eine Kennzeichnung der Kategorien und Sichtweisen, aus denen diese Anforderung entstanden ist.

Die für die Durchführung der Workshops verwendete Vorlage inklusive Kennzeichnung der einzelnen Schritte des Vorgehens ist in Bild 2 dargestellt.

In Abbildung 3 ist ein Beispiel der Rollen und Kategorien dargestellt. Darin wurden für die Beispielkategorien „Prozesssteuerung" und "Optimierung" aus Sicht der Beispiel-Rolle "Operator" jeweils ein „Job“ (gelb) mit zugehö- 


\begin{tabular}{|l|l|l|l|}
\hline $\begin{array}{l}\text { Job - Typische } \\
\text { Aufgabe, der Rolle }\end{array}$ & $\begin{array}{l}\text { Typische Probleme bei } \\
\text { der Erledigung des Jobs }\end{array}$ & $\begin{array}{l}\text { Wünsche, die die } \\
\text { Erledigung des Jobs } \\
\text { verbessern würden }\end{array}$ & $\begin{array}{l}\text { Welche Anforderungen } \\
\text { lassen sich daraus } \\
\text { ableiten? }\end{array}$ \\
\hline
\end{tabular}

\section{Unternehmenssicht}

Management
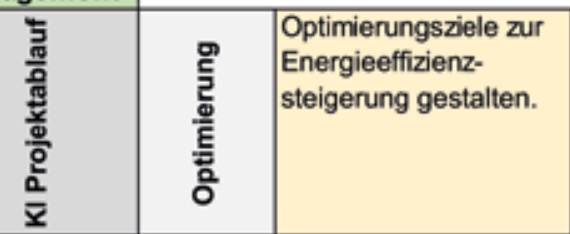

\begin{tabular}{|l|l|}
\hline Andere Aspekte wie & Wahl zwischen den \\
Produktqualität müssen & verschiedenen \\
neben der Energieeffizienz & Optimierungszielen (z.B. \\
ebenfalls geachtet & Energieeffizienz, \\
werden. Oft fehlt eine & Produktqualität, \\
Vergleichbarkeit. & Produktionsdauer).
\end{tabular}

Die Management braucht die Möglichkeit, zwischen Zielparametern von Energieeffizienz und Qualităt auswählen zu können

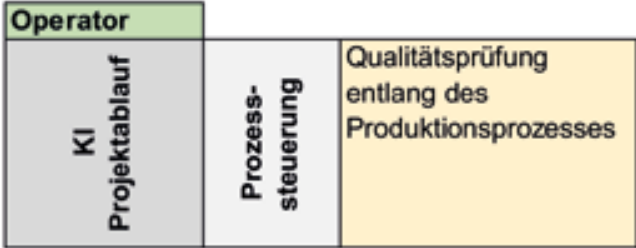

Parameter schwer zu
vergleichen, manchmal
sind Zusammenhänge
nicht bekannt.

Parameter schwer zu

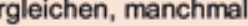
nicht bekannt.
Ampelsystem, um Übersichtlich sehen zu können, welche Produktionsläufe ungewöhnlich waren.
Der Operator benötigt eine Funktionalität für die Identifikation der Ursachen von energie-ineffizienten Produktionsläufen. rigem „Pain“ (rot) und "Gain“ (blau) aufgenommen. Die daraus entstehende Anforderung "weiß" resultiert aus den Eintragungen der vorherigen Spalten.

\section{Anwendung bei der Entwicklung einer Forschung- und Technologie- plattform}

Entwickelt, angewandt und evaluiert wird das beschriebene Vorgehen im Rahmen der Entwicklung einer Forschungs- und Technologieplattform zur Steigerung der Energieeffizienz, genannt „ecoKI". Diese Plattform wird von Forschungseinrichtungen entwickelt und soll im späteren Betrieb Unternehmen konfigurierbare KI- und Digitalisierungsbausteine zur Verfügung stellen. Im Fokus steht dabei die einfache und niedrigschwellige Anwendung dieser Technologien in der eigenen Produktion zur Steigerung der Energieeffizienz mit datengetriebenen Methoden. Auch die Wartbarkeit und einfache sowie schnelle Anpassung von bereitgestellten KI-Lösungen bei Veränderungen in der eigenen Produktion soll durch die Plattform unterstützt werden. Das Konzept sieht vor, dass die Menge an bereitgestellten Lösungen für generische Problemstellungen in Bezug auf das Thema Energie mit der Zahl der Anwendungen stetig weiter steigt. Eine Neuentwicklung, Weiterentwicklung und ein Feinschliff vorhandener Bausteine auf Basis der Anforderungen und Umsetzungen der nutzenden Industrieunternehmen sind Teil des Konzepts. In Abbildung 4 ist dieses grafisch dargestellt.

Im Rahmen der Anforderungserfassung für den Aufbau dieser Plattform werden Industrieunternehmen nach dem vorgestellten Vorgehen eingebunden. Zusätzlich wurden für vergange- ne Projekte in internen Workshops die Anforderungen dieser Projekte auf Basis der Erfahrungen und vorhandenen Dokumenten nachträglich abgebildet.

Die Details und Erfahrungen bei der Anwendung des Vorgehens werden im Folgenden für jeden Schritt dargelegt

\section{Identifizierung von Sichtweisen und Rollen}

Für die Anforderungserfassung der Plattform wurden alle Beteiligteneinbezogen. Es wurden folgende Rollen identifiziert

- „Entwickler*in“ der generischen Bausteine und der Plattform

- „Anwendungsingenieur*in“ für die Implementierung der Bausteine auf konkrete Problemstellungen bei einem Unternehmen

- „Anwender*in“ eines Unternehmens für die KI-Lösungen zur Steigerung der Energieeffizienz. Hier wurden außerdem die Sub-Rollen „Operator", „IT-Expert*in“ und „Manager" identifiziert.

Für die Anforderungserfassung der Plattform bilden diese Gruppen die wesentlichen Akteure ab. Aus Sicht der Autoren ist diese Unterteilung auch für andere KI- und Digitalisierungsprojekte sinnvoll. Natürlich können weitere Gruppen hinzugefügt werden.

\section{Definition der Kategorien}

Für die Kategorien wurden zwei Gruppen betrachtet. Zum einen der generische technische KI-Projektablauf mit den Kategorien „Prozesserfassung", "Datenintegration", "Modellierung", "Optimierung" und "Prozesssteuerung". Außerdem wurden übergreifende Themen betrachtet. Dazu zählen „IT-Sicherheit", „IT-Infrastruktur", „Wirtschaftlichkeit", ,Unabhängige Nutzbarkeit" und „Rechtliches".
Bild 3: Beispielhafte Einträge und erhobene Anforderungen im Rahmen des Vorgehens. 
Bild 4: Konzept der ecoKI-Plattform [13].
Literatur

[1] Brockhaus, C. P.; Bischoff, T. S.; Haverkamp, K.; Proeger, T.; Thonipara A.: Digitalisierung von kleinen und mittleren Unternehmen in Deutschland - ein Forschungsüberblick 2020.

[2] Christel, M. G.; Kang, K. C.: Issues in Requirements Elicitation. Fort Belvoir, Virginia, USA 1992

[3] Kotonya, G.; Sommerville, I.: Requirements engineering. Processes and techniques. In: Kotonya, G.; Sommerville, 1 . Worldwide series in computer science. Chichester, UK 1998.

[4] Berenbach, B.: Software \& systems requirements engineering. In: $B$ renbach, B. u. a. practice. New York USA 2009.

[5] Pacheco, C.; García, I.; Reyes, M. Requirements elicitation techniques: a systematic literature review based on the maturity of the techniques. In: IET softw. 4 (2018) S. 365-378.

[6] Farinha, C: da Silva, M. M:Requirements Elicitation with Web-Based Focus Groups. In: Pooley, J. (Hrsg): Information systems development. Reflections, challenges and new directions. New York, USA 2013.

[7] Ribeiro, C: Farinha, C.: Pereira, J.:Da Mira Silva, M.: Gamifying requirement elicitation: Practical implications and outcomes in improving stakeholders collaboration. In: Entertainment Computing 4 (2014) S. 335-345.

[8] Ordóñez, H.; Villada, A. F. E.;Vanegas, D. L. V.; Cobos, C.; Ordóñez, A.; Se govia, R. An Impact Study of Business Process Models for Requirements Elicitation in XP. In: Gervas (Hrsg): Computational Science and Its Applications, vol. 9155 (2015), S. 298-312.

[9] Castaneda, V:; Ballejos, L.C.; Caliusco, M. L; Galli, M.: The Use of Ontologies in Requirements Engineering. In: Global Journal of Research. In: Engineering vol. 10 (2010).

[10] Blomberg, J.; Giacomi, J.; Mosher, A Swenton-Wall, P: Ethnographic Field Methods and Their Relation to Design, S. 123-155.

[11] Sommerville, l.; Rodden, T.; Sawye P:; Bentley, R:; Twidale, M.: Integrating ethnography into the requirements engineering process. In: Proceedings of the IEEE (1992), S. 165-173.

[12] Clark, T.; Osterwalder, A.; Pigneur, Y: Business Model You. A One-Page Method For Reinventing You Career. Hoboken, New Jersey, USA 2012.

[13] ecoKl. Forschungs- und Technologieplattform: Steigerung der Energieeffizienz in der Produktion durch Digitalisierung und KI. URL: www. ecoki.de, Abrufdatum 28.09.2021

\section{Die ecoKI Plattform}

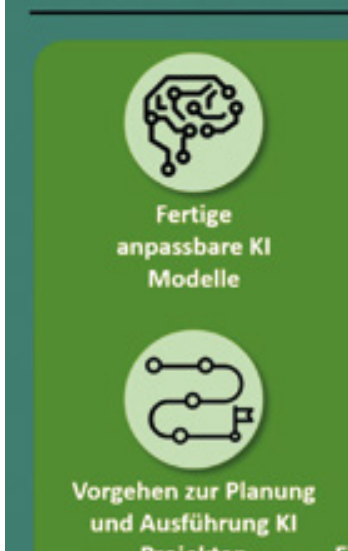

Projekten
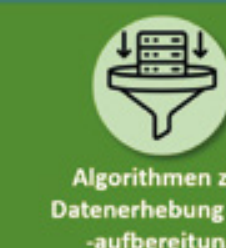

Algorithmen zur

Datenerhebung und

-aufbereitung

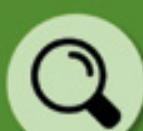

Methoden zur

Identifizierung von

Einsetzungspotential von $\mathrm{KI}$

zur Energieeffizienz
Der Mehrwert

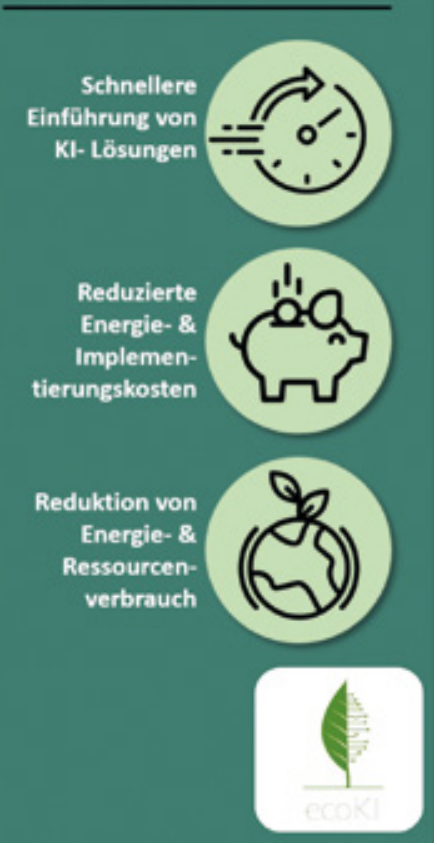

Nach Erfahrung der Autoren sind diese Kategorien ein guter erweiterbarer Startpunkt für die Anforderungsanalyse in KI- und Digitalisierungsprojekten.

\section{Durchführung der Workshops}

Das Konzept hat bisher zur Anforderungserfassung bei dieser Anwendung sehr gute Ergebnisse geliefert. An den Workshops nahmen jeweils zwei Mitglieder der Forschungseinrichtungen teil, die Moderation und Dokumentation der Ergebnisse übernahmen. Aus den Unternehmen nahmen jeweils mindestens drei Personen teil, die die unterschiedlichen Rollen im Unternehmen repräsentieren. Aufgrund der Coronavirus-Pandemie fand ein Großteil der Workshops online statt. Die Workshops dauerten 90 Minuten. Von den Teilnehmenden wurde das Feedback erhalten, dass sowohl die Kategorien als auch die Untergliederung in "Jobs" ",Pains" und "Gains" die Produktivität bei der Anforderungserfassung stark unterstützt haben.

\section{Ableitung der Anforderungen für die KI- und Digitalisierungs-Lösungen}

Die Anforderungen wurden nach den Workshops vom Moderationsteam gemeinsam abgeleitet. Auf Basis der Workshop-Dokumentation war dies für alle Anforderungen problemlos möglich.

\section{Teilen und Speichern der Anforderungen}

Die Anforderungen aus den Workshops wurden mithilfe des Ticketsystems „Redmine" eingepflegt und auf diese Weise für die nachfolgenden Entwicklungsschritte zur Verfügung gestellt.
Insgesamt waren die Erfahrungen mit diesem Vorgehen zur Erfassung von Anforderungen für die Entwicklung einer Plattform zur Steigerung der Energieeffizienz mit Digitalisierung und KI sehr positiv. Bisher wurden neun Projekte bzw. Untersuchungen in die Analyse eingebunden, die in derzeit etwa 130 validen Anforderungen resultierten. Die Anforderungsanalyse ist derzeit noch in der Durchführung und vollständige Ergebnisse sind erst nach Abschluss verfügbar.

Ausblick

In der Anwendung hat sich das entwickelte Vorgehen als sehr geeignet für die Aufnahme von Anforderungen für Digitalisierung- und KI-Projekte erwiesen. Als zukünftige weitere Verbesserung des Vorgehens werden derzeit zusätzliche Anforderungskategorien untersucht und evaluiert, die eine Relevanz für dieses Thema haben. Ein Beispiel dafür sind sozialwissenschaftliche Fragestellungen bezüglich der Akzeptanz von KI-Lösungen durch die Mitarbeitenden. Außerdem ist eine detaillierte Abstimmung des Moderationsvorgehens während des Workshops geplant.

Dieser Beitrag entstand im Rahmen des Projekts ecoKI „Entwicklung einer Forschungs- und Technologieplattform "Steigerung der Energieeffizienz mit Digitalisierung und KI'", das vom Bundesministerium für Wirtschaft und Energie unter dem Kennzeichen 03EN2047A gefördert wird.

\section{Schlüsselwörter:}

Künstliche Intelligenz, Machine Learning, Energieeffizienz, Digitalisierung, Nachhaltige Produktion, Requirements Engineering 